\title{
MAIN PROPERTIES OF THE HIPPARCOS CATALOGUE
}

\author{
F. MIGNARD \\ OCA/CERGA, Grasse, France
}

\begin{abstract}
The Hipparcos mission has come to a close with the end of the data processing on August 1996, only three years after the last observations were performed. About 118,000 stellar objects were observed and subsequently solved for their position, parallax and proper motion together with repeated determinations of their magnitude in the Hipparcos band. This paper outlines the main properties of the solution and explains how and when this information will be made available to the astronomical community.
\end{abstract}

\section{Introduction}

The ESA astrometric mission Hipparcos was the first space experiment fully dedicated to astrometry. The observation program came to its end in August 1993 after 37 months of effective observation which was followed by three more years of data analysis. The primary result is an astrometric catalogue of nearly 118,000 entries nearly evenly distributed over the sky with an astrometric accuracy of 1 mas or better for the brightest stars. This set of stars formed a pre-defined observing programme selected after a wide consultation of the astronomical community from their scientific interest and the possibility to allocate sufficient observational time to each of them. This task of preparing the stellar program was deemed as so challenging that it was entrusted by ESA to a dedicated Consortium, the Input Catalogue Consortium, with the goal of sorting out the 200 scientific proposals, amounting to more than 200,000 different stars, to obtain a working catalogue containing as many high priority objects as possible. The same group was also to coordinate the necessary new ground based observations so that the accuracy of the Input Catalogue meets the Hipparcos specifications. 


\section{The Hipparcos Mission}

The main scientific goals were those of astrometry :

- To provide a non-rotating celestial reference frame to which the motions of objects in the solar system or stars in the Galaxy could be referred

- To provide basic observational data for the studies of stellar properties within the Galaxy, such as distances, luminosities, masses and velocity vector.

The satellite was launched, by Ariane, in August 1989, and carried out 37 months of high quality observations before the mission was terminated following serious problems with the guidance system and the failure of the on-board computer in 1993. The satellite and the operational principles have been described in the literature and the interested reader will find technical information in the three volumes of the ESA SP-1111 report and in a special issue of Astronomy and Astrophysics dedicated to Hipparcos (Kovalevsky et al., Lindegren et al. 1992).

On a quantitative basis the initial objectives of the mission were to determine the five astrometric parameters of each program stars to a precision of 2 mas for the core of the catalogue at about 8.5 magnitude, along with the astrometry to 10-20 mas and two-color photometry for an additional 400,000 stars (the Tycho experiment). An optimized scanning law allowed to cover the whole sky more or less regularly in such a way that every star has been observed at 25 to 70 epochs according to its ecliptic latitude. Regarding the photometry the number of individual observations per star ranges between 70 to 300 , with again the ecliptic latitude as being the main factor of variation.

The Hipparcos Catalogue whose properties are described below, results from the merging of two largely independent solutions produced by scientists of the FAST (Fundamental Astrometry by Space Technic) and NDAC (Northern Data Analysis Consortium) consortia under the respective leadership of J. Kovalevsky (France) and L. Lindegren (Sweden). The treatment represents a major effort of more than 40 people extending over about 15 years between the design of the algorithms, the simulations and the processing of real data.

\section{Summary of the Astrometric Results}

A brief summary of the initial scientific goals is given in Table 1. The figures refer to the accuracy based on phase A and B assessments and on simulations carried out during the mission planning. Compared to the best available fundamental catalogues, this represented an improvement in accuracy of a factor 20 and nearly 100 in term of number of objects. 
The overall properties of the final catalogue are given in Table 2. The effective observation period is 37 months, slightly shorter than the time base, because of the occurrence of several interruptions in the observing program caused by hardware problems.

The Hipparcos epoch has been so chosen as to be close to the mean observation epoch for all the stars. The true mean epoch for each star may be different from the catalogue epoch by at most six months earlier or later. It was considered as most convenient to generate a catalogue with a single epoch, although this does not optimize the correlations between position and proper motions. The true mean epoch can be computed by searching for the time offset required to minimize the correlation between the right ascension and proper motion in right ascension, or that between the declination and proper motion in declination. These two epochs may differ by few weeks.

TABLE 1. The initial goals of the mission

\begin{tabular}{ll}
\hline Number of stars & 120,000 \\
Limiting magnitude & 12.5 \\
Positional accuracy & $2 \mathrm{mas}$ \\
Accuracy of parallax & $2 \mathrm{mas}$ \\
Accuracy of proper motions & $2 \mathrm{mas} / \mathrm{yr}$ \\
Mission duration & $2.5 \mathrm{yr}$ \\
\hline
\end{tabular}

TABLE 2. Overall properties of the catalogue

\begin{tabular}{ll}
\hline Measurement period & $1989.85-1993.21$ \\
Catalogue epoch & 1991.25 \\
Reference system & ICRS \\
Mean-Sky density & $\approx 3$ stars $/ \mathrm{deg}^{2}$ \\
Number of astrometric solutions & 117,955 \\
Systematic errors & $<0.1$ mas \\
\hline
\end{tabular}

In the Hipparcos program there was no direct observation of extragalactic object and the reference frames of the Hipparcos solutions were very consistent but had no reason to be rotation-free. Several radio stars were in the observing program and were also included in a VLBI program (Lestrade 
et al. 1994). A final rotation has allowed to align the final Hipparcos solution to the ICRS reference system. Therefore the Hipparcos Catalogue can been seen as the best available optical realization of the extragalactic reference frame.

\section{The Astrometric Results}

The main results of the absolute astrometry are summarized in Table 3 and in Figures 1-2. The major advance brought by Hipparcos is obviously the direct measurement of the trigonometric parallax for all the program stars, although for the most distant stars the parallax may have only a statistical, rather than individual, significance. The relative accuracy is more important in this respect than the fact the parallax may be known within 1 or 2 mas. With more than 20,000 stars measured with $\sigma(\pi) / \pi$ better than 10 percent, including several thousands binary stars, one can say that this is the start of a new era for the luminosity calibration and the mass determination. The number of doubles stars with good relative and absolute astrometry, including distances, is also an outstanding output of the mission.

The analysis of the Hipparcos parallaxes against a set of known very distant stars or against the best photometric parallaxes indicates that there is no systematic effect larger than 0.1-0.2 mas in the Hipparcos value.

TABLE 3. Summary of the Astrometric solution

\begin{tabular}{lll}
\hline & $\alpha \cos \delta$ & $\delta$ \\
Median precision of positions (Hp <9) (mas) & 0.77 & 0.64 \\
Median precision of proper motions (Hp <9)(mas/yr) & 0.88 & 0.74 \\
\hline Median precision of parallaxes $(\mathrm{Hp}<9)(\mathrm{mas})$ & 0.97 \\
Number of stars with $\sigma(\pi) / \pi<0.1$ & 20,800 \\
Number of stars with $\sigma(\pi) / \pi<0.2$ & 50,000 \\
\hline Number of double star systems & \\
Range of separations (arcsec) & 12,195 \\
Range of magnitude difference & $0.1-25$ \\
Median accuracy of the separation (mas) & $0.0-3.5$ \\
Nuumber of suspected astrometric binaries & 11 & 2,900 \\
& \\
\hline
\end{tabular}

The median accuracy in Table 3 is typical for the bulk of the catalogue for stars of magnitude 8 to 8.5 . The variation of the mean accuracy of 

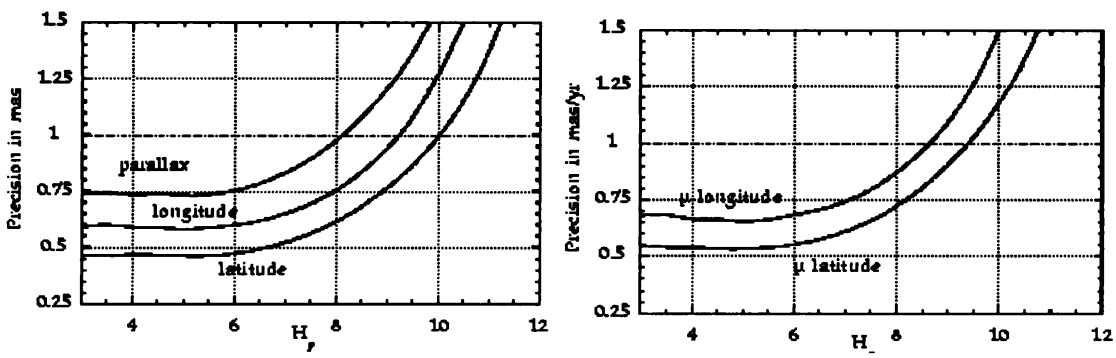

Figure 1. Precision of the astrometric parameter in the final Hipparcos solution as a function of the magnitude $H p$.
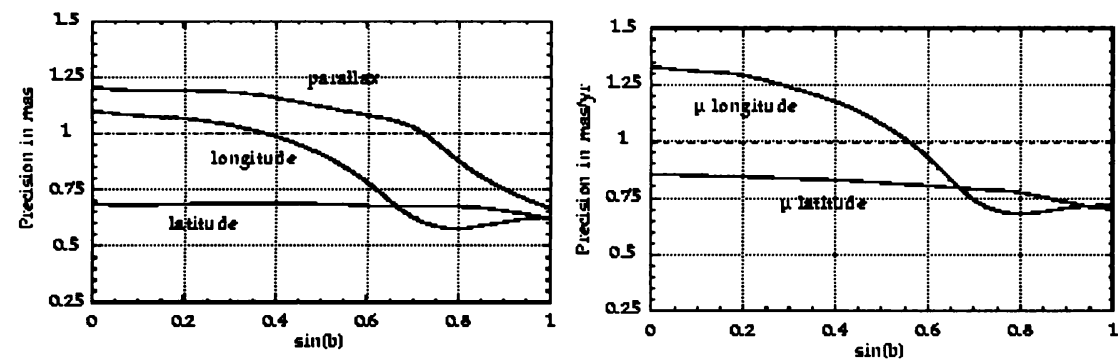

Figure 2. Precision of the astrometric parameter in the final Hipparcos solution as a function of the ecliptic latitude

the five astrometric parameters as a function of the magnitude is shown in Figure 1. At the faint end the photon noise was the limiting factor and the diagram gives the expected increase in the standard error with the magnitude. For stars brighter than 8 mag, the accuracy does not improve as expected, as the limiting factor is chiefly instrumental and weakly sensitive to the magnitude. The difference between the accuracy of longitude and latitude comes as no surprise and is a mere consequence of the geometry of the scanning. Besides the brightness, one must add that the accuracy depends strongly on the ecliptic latitude, as illustrated in Figure 2 for the same parameters. There is virtually no effect in latitude, but a very conspicuous one in longitude, with about a factor two between the standard error for ecliptic stars compared to polar stars. The minimum at about $b \approx 47$ degrees follows again from the scanning law, with the axis of rotation of the satellite kept at 43 degrees from the direction of the Sun.

\section{The Results of the Photometric Treatment}

Although the Hipparcos mission was primarily planned and optimized to produce good astrometric measurements, it was soon realized that the sig- 
nal conveyed much information on the star brightness, provided a good monitoring of the instrument sensitivity was undertaken.

At each grid crossing a magnitude was determined for all programme stars in a well defined photometric system, kept constant during the mission. Altogether the missions ends up with $13 \times 10^{6}$ such measurements spread over 118,200 stars, that is to say an average of 110 magnitude determinations for each star, with an accuracy of 0.01-0.02 mag for a typical Hipparcos star of magnitude 8.5. This constitutes the most comprehensive photometric survey so far, combining both precision and homogeneity of the photometric system all over the sky. Summary figures are given in Table 4.

TABLE 4. Summary of the photometric solution

\begin{tabular}{ll}
\hline Number of entries with solution & 118,204 \\
Photometric band & $H p$ \\
$\lambda_{\text {eff }}$ & $550 \mathrm{~nm}$ \\
Photometric accuracy for one observation $(H p=8.5)$ & $0.012 \mathrm{mag}$ \\
Average number of observations per star & 110 \\
Total number of photometric observations & $13 \times 10^{6}$ \\
Median photometric accuracy $(H p<9)$ & $0.0015 \mathrm{mag}$ \\
Number of variables detected & 6,000 \\
Number of suspected variables & 5,700 \\
& \\
\hline
\end{tabular}

The number of variables detected or suspected depends on various statistical thresholds set in the variability analysis. Although this was done with great care and based on several statistical tests, it may happen that further analyses based on the Hipparcos data and additional ground based data could end with different figures.

The actual distribution of the standard errors of the accumulated photometry (the standard error of the median of the $\approx 110$ measurements of a star) is shown in Figure 3. All the constant bright stars are on the left of the diagram with $\sigma(H p)<0.001$ mag. The rightmost column must be associated with the variable stars, since the scatter among the individual observations is large as a result of the variability. The left diagram gives the accuracy at the transit level, that is to say for the individual observations. 

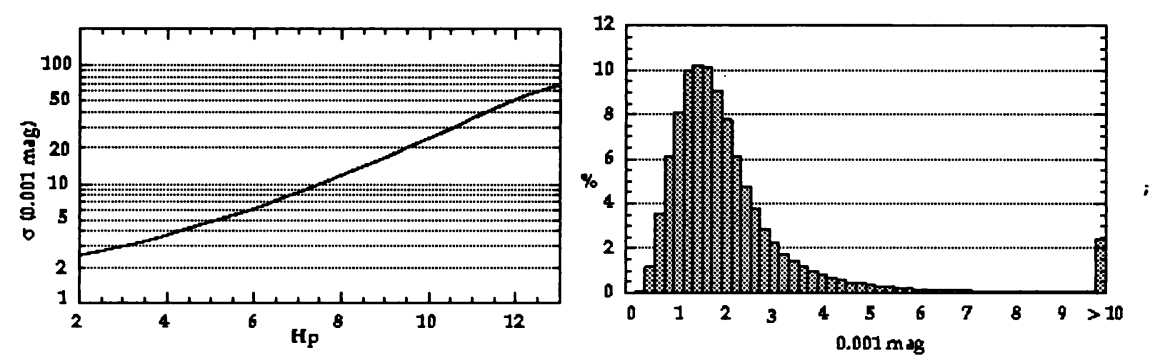

Figure 3. Precision of the photometric solution in the Hipparcos Catalogue. Left : Variation of the precision of the photometric accuracy as a function of the magnitude. Right : The distribution of the standard errors in the accumulated photometry

\section{Conclusion}

The Hipparcos final product will comprise 16 printed volumes, a set of CD-ROMs with the Hipparcos and Tycho Catalogues in ASCII files, and a second set including all the files in binary form along with a dedicated software permitting interrogation, sample constructions on any of the fields or their logical combination, and graphic display. The availability to the general astronomical community in due for June 1997. Advanced data release was granted to PIs and early proposers respectively in August 1996 and January 1997 for approved scientific programs. The first scientific results based on the exploitation of the Hipparcos data, will be presented at a meeting to be held in May 1997 in Venice.

\section{Acknowledgements}

This short overview reflects under the name of a single author the full dedication of many scientists, engineers and technicians who have devoted most of their professional activity to the project during the last fifteen years. Their name and affiliation will appear in the Hipparcos documentation. They are here collectively thanked for their invaluable contribution.

\section{References}

Kovalevsky J. et al. 1992, The FAST Data Analysis Consortium : Overview of the reduction software, Astron.Astrophys., 258, 326.

Lestrade, J. F., Jones, D.L. et al. 1995, Astron.Astrophys., 304, 182.

Lindegren L. et al. 1992, The NDAC Data Analysis Consortium : Overview of the reduction methods, Astron.Astrophys., 258, 18. 